\title{
P02-38
}

\section{ANHEDONIA AT PSYCHIATRIC IN-PATIENTS}

N. Orlova, M. Shkliar

Kiev City Clinical Psychoneurologycal Hospital, Kiev, Ukraine

Objectives: Anhedonia - loss of the capacity to experience pleasure, the inability to gain pleasure from normally pleasurable experiences - is a core clinical feature of some psychiatric illnesses and very important symptom of many somatic disorders. Its classification and treatment are very actual problems of modern psychiatry.

Methods: For assessment anhedonia level at psychiatry in-patients we used the "Scale for physical and social anhedonia" Chapman L\&J.

Results: 279 psychiatry in-patients with mental illnesses (Schizophrenia, Mood disorders and etc.) (174 men, 105 women) with a mean age of $36,42( \pm 0,9)$ and 123 healthy controls $(75$ men, 48 women) with a mean age of $36,49( \pm 1,8)$ were examine by this scale. $50,54 \%$ cases $(n=141)$ had anhedonia $(p \leq 0,05)$. At patients with diagnostic category $298(D S M-I V-T R)$ anhedonia was in $67 \%$ cases $(n=93)$, diagnostic category 296 (DSM-IV- TR) anhedonia was in $21 \%$ cases $(n=31)$. Other diagnostic categories DSMIV-TR were insignificant. At healthy controls anhedonia was in $21,1 \%$ cases $(n=26)$. The factor analysis was selected subtypes of anhedonia in mental illnesses. We studied the correlation of level anhedonia and prolactin level in blood at patients who had treatment by atypical antipsychotic. This correlation was significant $(p \leq 0,05)$.

Conclusion: Results of analyze the modern literature and owner data may tell us about pathogenetics subtypes of anhedonia. There are: in depression, in schizophrenia and in hyperprolactinemia. This clinical classification may ability for effective treatment these patients. 